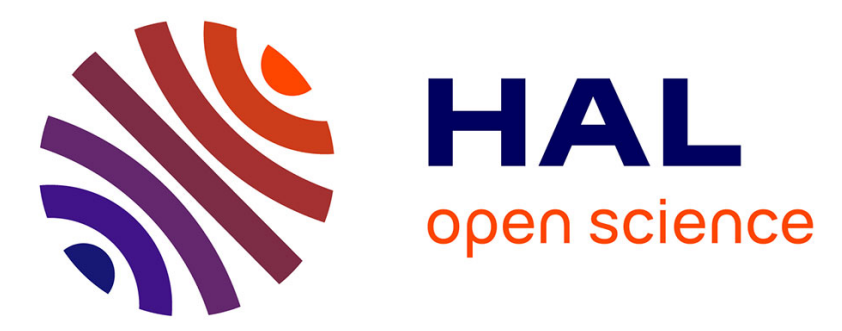

\title{
Natural polymeric 3-alkylpyridinium salt affects vertebrate skeletal muscle contractility by preferentially blocking neuromuscular transmission
}

Monika Cecilija Žužek, Marjana Grandič, Evelyne Benoit, Robert Frangež

\section{- To cite this version:}

Monika Cecilija Žužek, Marjana Grandič, Evelyne Benoit, Robert Frangež. Natural polymeric 3alkylpyridinium salt affects vertebrate skeletal muscle contractility by preferentially blocking neuromuscular transmission. Toxicology Letters, 2017, 281, pp.95-101. 10.1016/j.toxlet.2017.09.007. hal-01661797

\section{HAL Id: hal-01661797 \\ https://hal.science/hal-01661797}

Submitted on 21 May 2020

HAL is a multi-disciplinary open access archive for the deposit and dissemination of scientific research documents, whether they are published or not. The documents may come from teaching and research institutions in France or abroad, or from public or private research centers.
L'archive ouverte pluridisciplinaire HAL, est destinée au dépôt et à la diffusion de documents scientifiques de niveau recherche, publiés ou non, émanant des établissements d'enseignement et de recherche français ou étrangers, des laboratoires publics ou privés. 
1 Natural polymeric 3-alkylpyridinium salt affects vertebrate skeletal muscle 2 contractility by preferentially blocking neuromuscular transmission

3

4

5

6

7

8

9

\title{
Monika Cecilija Žužek ${ }^{a}$, Marjana Grandič ${ }^{b}$, Evelyne Benoit ${ }^{c}$ and Robert Frangeža
}

${ }^{a}$ Institute of Preclinical Sciences, Veterinary Faculty, University of Ljubljana, Gerbičeva 60, 1000 Ljubljana, Slovenia

${ }^{\mathrm{b}}$ Institute of food safety, feed and environment, Department of environment, animal nutrition, welfare and hygiene, Veterinary Faculty, Universitiy of Ljubljana, Cesta v Mestni log 47, 1000 Ljubljana, Slovenia

${ }^{\mathrm{c} D R F / I n s t i t u t}$ des Sciences de la Vie Frédéric Joliot/SIMOPRO, CEA de Saclay, and Institut des Neurosciences Paris-Saclay (Neuro-PSI), UMR 9197 CNRS/Université Paris-Sud, 91190 Gif-sur-Yvette, France

*corresponding author: Robert Frangež; E-mail address: robert.frangez@vf.uni-lj.si; tel.: 0038614779131

\section{Highlights}

- nPoly-3-APS preferentially blocks the skeletal neuromuscular transmission

- nPoly-3-APS acts by a non-depolarizing mechanism

- In vivo toxicity of nPoly-3-APS mainly occurs through antagonist action on nAChRs

\begin{abstract}
The effects of natural polymeric alkylpyridinium salt (nPoly-3-APS), a potent acetylcholinesterase inhibitor isolated from the marine sponge Reniera sarai, were studied on isolated mouse phrenic nerve-hemidiaphragm muscle preparations using electrophysiological approaches. nPoly-3-APS inhibited nerve-evoked isometric muscle twitch and tetanic contraction in a concentration-dependent manner $\left(\mathrm{IC}_{50}=29.4 \mu \mathrm{M}\right.$ and $18.5 \mu \mathrm{M}$, respectively) and produced a $30-44 \%$ decrease of directly muscle-elicited twitch and tetanus amplitudes at $54.4 \mu \mathrm{M}$. Additionally, nPoly-3-APS (9.1-27.2 $\mu \mathrm{M})$ markedly decreased the amplitude of miniature endplate potentials, while their frequency was only affected at the highest concentration used. Endplate potentials were also inhibited by nPoly-3-APS in a concentration-dependent manner $\left(\mathrm{IC}_{50}=20.1 \mu \mathrm{M}\right)$, without significant change in the resting membrane potential of muscle fibers (up to $54.4 \mu \mathrm{M}$ ). In conclusion, our results show, for the first time, that nPoly-3-APS preferentially blocks the neuromuscular transmission, in vitro, by a non-depolarizing mechanism. This strongly suggests that the in vivo toxicity of nPoly-3APS mainly occurs through an antagonist action of the compound on nicotinic acetylcholine receptors of skeletal muscles.
\end{abstract}

Key words: Natural Poly-APS; Neuromuscular junction; AChE inhibitors; Motor endplate potential; Skeletal muscle contraction 


\section{Introduction}

Natural polymeric 3-alkylpyridinium salt (nPoly-3-APS), isolated from the marine sponge Reniera sarai (Sepcic et al., 1997), shows a broad spectrum of biological activities, including antibacterial and antifouling activities, acetylcholinesterase (AChE) inhibition, cytotoxicity, as well as hemolysis and selective toxicity against non-small cell lung cancer (NSCLC) tumor cells in vitro and in vivo (Sepcic et al., 1997; Sepcic et al., 1998; Malovrh et al., 1999; Faimali et al., 2003; Tucker et al., 2003; Chelossi et al., 2006; Paleari et al., 2006; Catassi et al., 2008; Elersek et al., 2008). nPoly-3-APS can also be used for the stable transfection of nucleated mammalian cells (Tucker et al., 2003). Its likely application in medicine led to the synthesis of a series of analogs with different degree of polymerization and length of alkyl chains (Houssen et al., 2010). Synthetic analogs of Poly-3-APS, such as APS12-2, APS3 (Grandic et al., 2011; Grandic et al., 2013), APS8 (unpublished data) and structurally related compounds, are promising new chemotherapeutic agents that exhibit very low in vivo toxicity in experimental animals. Recently, the effects of some of these synthetic analogs possessing potent AChE-inhibitory properties were studied on neuromuscular transmission in skeletal muscle (reviewed in Grandic and Frangez, 2014) to avoid unwanted peripheral side effects that could appear in patients treated with some AChE inhibitors. APS12-2 acts as a potent non-competitive AChE inhibitor with an inhibitory constant $\left(K_{i}\right)$ of $34 \times 10^{-3} \mathrm{nM}$ (Houssen et al., 2010). It exerts hemolytic, antibacterial and antifungal actions (Houssen et al., 2010). Some nicotinic cholinergic antagonists, such as APS3 and APS8, have shown promise in lung cancer treatment because of their selective cytotoxicity against NSCLC, the most common form of lung cancer (Zovko et al., 2013). NSCLC cells express cholinergic signaling mediators, including neuronal-type $\alpha 7$-nicotinic acetylcholine receptors (Paleari et al., 2006; Catassi et al., 2008). The inhibitory effects of the synthetic analogs APS12-2 and APS-3 on neuromuscular transmission and nerve-evoked skeletal muscle contraction were previously reported (Grandic et al., 2012; Grandic et al., 2013). It was demonstrated that the neuromuscular blockade produced by these two compounds is due to their direct inhibitory effect on muscle-type nicotinic acetylcholine receptors (nAChRs). Therefore, it was of interest to determine whether the natural compound nPoly-3-APS shares the same biological effects on neuromuscular transmission and skeletal muscle functioning. In particular, due to the structural similarity between nPoly-3-APS and quaternary ammonium compounds that have been reported to block muscle-type nAChRs, the aim of this study was to investigate the effects and underlying mechanisms of the natural compound on neuromuscular transmission and skeletal muscle contraction. We show, for the first time, that nPoly-3-APS affects the skeletal muscle functioning, in vitro, by preferentially blocking the neuromuscular transmission probably through an antagonist action on muscle-type nAChRs.

\section{Materials and methods}

\subsection{Materials}

\subsubsection{Drugs}

nPoly-3-APS was isolated from the marine sponge Reniera sarai according to Sepčić et al. (1997). Before use, the toxin was dissolved in a sterile $0.9 \%$ saline solution at a stock 
concentration of $10 \mathrm{mg} / \mathrm{mL}$ and was stored at $4^{\circ} \mathrm{C}$. Neostigmine methyl sulfate (Tokyo Chemical Industry CO./LTD, Japan), 3,4-diaminopyridine (3,4-DAP; Sigma-Aldrich, USA) and $\mu$-conotoxin GIIIB (Bachem, Switzerland) were of the highest grade available.

\subsubsection{Experimental animals and neuromuscular preparations}

Adult male Balb/C mice were obtained from the animal breeding facility at the Veterinary Faculty (University of Ljubljana, Slovenia). All the experiments followed ethical standards and were approved by the administration of the Republic of Slovenia for food safety, veterinary and plant protection with permit no. 34401-20/2009/30.

Mice were sacrificed by cervical dislocation followed by immediate exsanguination. The diaphragm muscle along with both phrenic nerves was dissected and split into two neuromuscular preparations. Each hemidiaphragm was maintained in oxygenated standard Krebs-Ringer solution composed of the following: $154 \mathrm{mM} \mathrm{NaCl}, 5 \mathrm{mM} \mathrm{KCl}, 2 \mathrm{mM} \mathrm{CaCl} 2,1$ $\mathrm{mM} \mathrm{MgCl} 2,5 \mathrm{mM}$ HEPES and $11 \mathrm{mM}$ D-glucose (pH 7.4). All experiments were performed at $22-24^{\circ} \mathrm{C}$.

\subsection{Methods}

\subsubsection{Muscle twitch and tetanus recordings}

The hemidiaphragm was pinned to the organ bath on its lateral side. It was connected to an isometric mechano-electrical transducer (Grass Instruments, West Warwick, RI, USA) by silky thread via a stainless-steel hook, attached to its tendinous part. The motor nerve of the isolated neuromuscular preparation was stimulated using a square pulse S-48 stimulator (Grass Instruments, West Warwick, RI, USA) via a suction electrode with pulses of $0.1 \mathrm{~ms}$ and supramaximal voltage (typically 5-10 V) applied at a $0.1-\mathrm{Hz}$ frequency. Direct muscle stimulation was evoked by electric field stimulation via a platinum electrode assembly in the organ bath connected to the S-48 Grass stimulator and producing pulses of $0.1 \mathrm{~ms}$ and 50-120 $\mathrm{V}$ applied at a $0.1-\mathrm{Hz}$ frequency. Directly- (via muscle stimulation) or indirectly- (via nerve stimulation) evoked tetanic contraction was obtained with a pulse train duration of $1000 \mathrm{~ms}$ and a stimulation rate of $80 \mathrm{~Hz}$. To achieve the maximal contractile response upon nerveevoked stimulation, the stable resting tension for each neuromuscular preparation (typically 1.5-2.5 g) was adjusted approximately $20 \mathrm{~min}$ before starting the experiment. Muscle twitch or tetanic tension was measured using a Grass FT03 force transducer (Grass Instruments, West Warwick, RI, USA). Electrical signals were amplified using a P122 strain gauge amplifier (Grass Instruments, West Warwick, RI, USA) and digitized using a data acquisition system (Digidata 1440A; Molecular Devices, Sunnyvale, CA, USA) at a sampling rate of 1 $\mathrm{kHz}$. The inhibitory response of nPoly-3-APS was continuously measured during 60-90 min after compound application. The muscle twitch or tetanic tension blockade produced by nPoly-3-APS was expressed as the percentage of the maximal response recorded before compound application. The concentrations of nPoly-3-APS used were 9.1, 18.1, 27.2, 36.2, 45.3 and $54.4 \mu \mathrm{M}$.

\subsubsection{Membrane potential recordings}

Hemidiaphragm preparations were equilibrated for $30 \mathrm{~min}$ in standard Krebs-Ringer solution added with 1.6 $\mu \mathrm{M} \mu$-conotoxin GIIIB, which blocks Nav1.4 muscle sodium channel subtypes 
(Cruz et al., 1985; Hong and Chang, 1989) and allows the recording of the full-sized amplitude of the endplate potentials (EPPs). Miniature endplate potentials (MEPPs), EPPs and the resting membrane potential $\left(\mathrm{rV}_{\mathrm{m}}\right)$ were recorded from superficial hemidiaphragm muscle fibers using intracellular borosilicate glass microelectrodes and Axoclamp 900A microelectrode amplifier (Molecular Devices, Sunnyvale, CA, USA). Microelectrodes were pulled using a P-97 Flaming/Brown micropipette puller (Sutter Instruments, Novato, CA, USA) and were filled with $3 \mathrm{M} \mathrm{KCl}$ solution. Only microelectrodes with resistance of 10-20 $\mathrm{M} \Omega$ were used. Endplate regions, where EPPs were recorded, were indicated by the presence of MEPPs. EPPs were evoked by stimulating the phrenic nerve via the bipolar suction electrode with supramaximal square pulses of $0.1 \mathrm{~ms}$ at $0.5 \mathrm{~Hz}$ using the S-48 Grass stimulator. MEPPs and EPPs were digitized at $25 \mathrm{kHz}$ using Digidata 1440A and pCLAMP 10 (Molecular Devices, Sunnyvale, CA, USA) and were stored for later analysis using pCLAMP-Clampfit 10 software (Molecular Devices, Union City, CA, USA). The recordings were performed before treatment, 45 and 90 min after the application of nPoly-3-APS and 15 min after the compound wash-out. MEPP and EPP amplitudes were normalized to a $\mathrm{rV}_{\mathrm{m}}$ of $70 \mathrm{mV}$ using the formula $\mathrm{V}_{\mathrm{c}}=\mathrm{V}_{\mathrm{o}} \mathrm{x}(-70) / \mathrm{rV}_{\mathrm{m}}$, where $\mathrm{V}_{\mathrm{c}}$ is the normalized amplitude of MEPPs and EPPs, and $\mathrm{V}_{\mathrm{o}}$ is their recorded amplitude (Pardo et al., 2006). The concentrations of nPoly-3-APS used were 1.8, 9.1, 18.1, 27.2, 36.2 and $54.4 \mu \mathrm{M}$.

\subsubsection{Data analysis and statistics}

The results are presented as the means \pm S.E. The nPoly-3-APS concentrations producing $50 \%$ inhibition of initial responses $\left(\mathrm{IC}_{50}\right)$ were determined by fitting the concentrationresponse relationships using the four-parameter nonlinear regression model (GraphPad Prism version 6.00). The data were statistically analyzed using Sigma Plot for Windows version 12.5 (Systat Software Inc., USA). Student's two-tailed $t$-test was used for statistical analysis of the data. Statistical significance was set at a $P$ value $\leq 0.05$.

\section{Results}

\section{1 nPoly-3-APS blocks muscle contraction in vitro}

The effects of natural nPoly-3-APS (9.1-54.4 $\mu \mathrm{M})$ were first determined on nerve-evoked and directly muscle-elicited isometric twitch and tetanic contraction in mouse hemidiaphragm preparations. A representative example of the time-course of nPoly-3-APS effects on muscle contraction is shown in Fig. 1A. The compound $(36.2 \mu \mathrm{M})$ blocked nerve-evoked twitch and tetanic contraction within $90 \mathrm{~min}$ (Fig. 1A and B). This effect was in part reversible since, after about $30 \mathrm{~min}$ of thorough washing of the preparation with standard physiological solution, the nerve-evoked muscle twitch and tetanic contraction were restored partially (Fig. 1A). In contrast, directly muscle-elicited twitch and tetanic contraction were much less affected by a similar compound concentration (Fig. 1A and C). The nPoly-3-APS-induced block of muscle contraction occurred in a concentration-dependent manner (Fig. 1D and E). The $\mathrm{IC}_{50}$ values, calculated from the concentration-response curves, were 18.5-29.4 $\mu \mathrm{M}$ for nerve-evoked muscle contraction, and $56-90 \mu \mathrm{M}$ for directly muscle-elicited contraction (Fig. 1E). Therefore, nPoly-3-APS was more than 3 times more efficient to block nerve-evoked than directly muscle-elicited twitch and tetanic contraction. It is worth noting that no decrease 
of muscle contraction amplitude occurred during 90-min control experiments during which neuromuscular preparations were only bathed with Krebs-Ringer solution (data not shown).

\section{Figure 1 near here}

During the development of the contraction block, obtained 30 min after the application of $36.2 \mu \mathrm{M}$ nPoly-3-APS (i.e. $33 \pm 4.4 \%, \mathrm{n}=4$ ), $3 \mu \mathrm{M}$ neostigmine, which is known to inhibit AChE (Alderdice, 1982), did not modify muscle twitch and tetanic contraction (data not shown). Additionally, $300 \mu \mathrm{M}$ 3,4-diaminopyridine, a well-known potassium channel blocker in nerve terminals (Molgó et al., 1980), significantly increased the twitch and tetanic contraction amplitudes to approximately $120 \pm 11 \%(n=4)$ of their initial value (data not shown).

\subsection{Effects of nPoly-3-APS on $r V_{m}$, MEPPs and EPPs}

In a second set of experiments, we tested whether nPoly-3-APS has some effects on $\mathrm{rV}_{\mathrm{m}}$, MEPPs and EPPs since the compound was shown to block preferentially nerve-evoked muscle twitch and tetanic contraction.

\subsection{1 nPoly-3-APS does not affect $r V_{m}$}

nPoly-3-APS (1.8-54.4 $\mu \mathrm{M})$, i.e. at concentrations that partially or completely block nerveevoked muscle twitch and tetanic contraction in mouse hemidiaphragms (see Fig. 1D and E), did not significantly affect the $\mathrm{rV}_{\mathrm{m}}$ of muscle fibers recorded in the endplate regions of the surface fibers after 45 and 90 min of compound application (Fig. 2). In particular, the mean $\mathrm{rV}_{\mathrm{m}}$ was $-65.5 \pm 1.7 \mathrm{mV}$ under control conditions (i.e. at time zero, before compound application), and $-64.3 \pm 1.5 \mathrm{mV}$ and $-61.2 \pm 0.6 \mathrm{mV}, 45$ and $90 \mathrm{~min}$ after exposure to 54.4 $\mu \mathrm{M}$ nPoly-3-APS, respectively.

\section{Figure 2 near here}

\subsection{2 nPoly-3-APS reduces MEPP and EPP amplitudes}

The effects of nPoly-3-APS on the MEPP amplitude and frequency are shown in Fig. 3. nPoly-3-APS (9.1-27.2 $\mu \mathrm{M})$, in a concentration-dependent manner, significantly reduced the amplitude of MEPPs recorded in superficial muscle fibers of the mouse hemidiaphragm (Fig. $3 \mathrm{~A}$ and $\mathrm{B}$ ), without significant decrease of their frequency except at the highest compound concentration (Fig. 3C). At higher concentrations (36.2-54.4 $\mu \mathrm{M}$ ), nPoly-3-APS completely abolished MEPPs (Fig. 3A). No significant change in the EPP half-decay time was observed among the concentrations studied (Fig. 3A).

\section{Figure 3 near here}

Electrophysiological recordings of EPPs from the endplates of the mouse hemidiaphragm exposed to nPoly-3-APS $(1.8-54.4 \mu \mathrm{M})$ for 90 min revealed that the compound reduced their amplitude in a concentration-dependent manner (Fig. 4). The $\mathrm{IC}_{50}$ calculated value for the nPoly-3-APS-induced reduction of the EPP amplitude was $20.1 \mu \mathrm{M}$ (Fig. 4B). No significant change in the EPP half-decay time was observed among the concentrations studied (Fig. 4A).

Figure 4 near here 


\section{Discussion}

This study shows, for the first time, the effects of natural alkylpyridinium polymer (nPoly-3APS), produced by the marine sponge Reniera sarai, on neuromuscular transmission and skeletal muscle functioning. In a previous study, it was shown that nPoly-3-APS after i.v. administration at a sublethal dose to male Wistar rats increased the residual respiratory volume, prolonged expirium, produced bradypnea; at a lethal dose, nPoly-3-APS causes respiratory arrest (Bunc et al., 2000). However, nPoly-3-APS showed many interesting biological activities (reviewed in Turk et al., 2007). Its application in medicine led to the synthesis of a series of synthetic analogs with different degrees of polymerization and different lengths of alkyl chains (Houssen et al., 2010). Recently, the effects of some of these synthetic analogs due to their AChE inhibitory potency were studied on the skeletal neuromuscular transmission (reviewed in Grandic and Frangez, 2014). Experiments on skeletal neuromuscular preparations revealed that APS12-2, a non-competitive AChE inhibitor which was previously shown to be selectively cytotoxic toward NSLC cells in vitro, is a potent antagonist of skeletal muscle nAChRs (Grandic et al., 2012). Later, it was shown that the synthetic polymeric 3-alkylpyridinium salt (APS3) in vitro inhibits nerve-evoked muscle contraction of the mouse hemidiaphragm due to specific block of muscle-type nAChRs with an $\mathrm{IC}_{50}$ of $20.3 \mu \mathrm{M}$ (Grandic et al., 2012). Therefore, it was of interest to examine the natural molecule nPoly-3-APS for similar biological effects on skeletal neuromuscular transmission and to propose different mechanistic hypotheses to explain its underlying molecular mechanism of action.

Our results obtained from phrenic nerve-hemidiaphragm preparations are summarized in Table 1. They show that nPoly-3-APS, at concentrations ranging from 9.1 to $54.4 \mu \mathrm{M}$, progressively blocked the maximal amplitude of nerve-evoked isometric muscle twitch, sustained (tetanic) contraction and EPPs, without any noticeable change in their decay phase. Additionally, relatively small decrease of maximal amplitude was observed on directly muscle-elicited twitch and tetanic contraction. The natural compound, without notably affecting the frequency (except a reduction observed in the presence of the highest compound concentration) and the decay phase, decreased the MEPP amplitude measured in hemidiaphragm superficial muscle fibers. Taking into account that the muscle fiber $\mathrm{rV}_{\mathrm{m}}$ remained unchanged even after the longest exposure to the highest concentration of nPoly-3APS used, these results indicate a preferential postsynaptic activity of the natural compound. Indeed, they are exactly what is expected if nPoly-3-APS produces muscle-type nAChR blockage (Table 1). This mechanistic hypothesis was previously proposed to explain the effects of APS12-2 and APS3, two synthetic analogs of polymeric alkylpyridinium salts isolated from Reniera sarai, which were similar to those of the natural compound (Grandic et al., 2012; Grandic et al., 2013). Additionally, available information in the literature indicates that 3-alkylpyridinium polymers from the marine sponge Reniera sarai, at approximatively 200-2000 times lower concentrations than those used in the present study, possess strong AChE activity, and a detailed mechanism of kinetics of AChE inhibition has been proposed (Sepcic et al., 1997; Sepcic et al., 1998; reviewed in Turk et al., 2007). The lack of AChE activity at the neuromuscular junction level allows acetylcholine (ACh) to accumulate in the synaptic cleft and thus to activate repetitively nAChRs (Van der Kloot et al., 1994). It is characterized, in particular, by muscle inability to sustain a tetanic contraction under 
repetitive high frequency stimulation of the motor nerve, due to both ACh accumulation and decreased $\mathrm{rV}_{\mathrm{m}}$, by enhancement of nerve-evoked muscle twitch, EPP and MEPP amplitudes, as well as MEPP frequency, and by prolongation of their decay phase (Table 1; Chang et al., 1986; Katz and Miledi, 1973). However, no physiological evidence was obtained in the present study of an eventual anti-AChE effect of nPoly-3-APS since we did not observe any of these AChE inhibition characteristics (Table 1). Furthermore, the nerve-evoked muscle twitch block was not antagonized/augmented by $3 \mu \mathrm{M}$ neostigmine, which almost completely inhibits AChE in the neuromuscular junctions of the mouse hemidiaphragm (Minic et al., 2003). Possible involvement of anti-AChE activity of the natural compound is therefore excluded in the present results. Different hypotheses can be proposed to explain the absence of an observation of nPoly-3-APS-induced AChE inhibition at the level of mouse skeletal neuromuscular junction. The first is that such an inhibition is masked by the nPoly-3-APSproduced blockage of muscle-type nAChRs. However, none of the AChE inhibition characteristics were observed at low concentrations $(1.8-9.1 \mu \mathrm{M})$ which produced no marked nAChR blockage even if they were 100-1000 times higher than those reported previously to inhibit AChE (Sepcic et al., 1997). The second hypothesis is the polycationic nature of nPoly3-APS, leading to limited ability of the natural compound to reach its binding site since its AChE activity was previously shown to occur only in aqueous solutions (Sepcic et al., 1997), which may also have an impact on its activity in biological systems. Finally, it is worth noting that these authors reported only inhibition of recombinant insect, electric eel and human erythrocyte $\mathrm{AChE}$, as well as equine BuChe, without any information on mammal neuronal AChE. Knowing that there is low percentage of similarity between mammal (human and mouse), insect and fish AChE amino-acid sequences (Wiesner et al., 2007), one cannot exclude that the affinity of nPoly-3-APS for mouse AChE is very low compared to that for mouse muscle-type AChRs.

\section{Table 1 near here}

The partial block of nerve-evoked muscle twitch produced by nPoly-3-APS was completely reversed by the potassium channel blocker 3,4-diaminopyridine, known to prolong the duration of action potential, to increase both $\mathrm{Ca}^{2+}$ entry into nerve terminals and ACh release from motor terminals (Molgỏ et al., 1980; Hong and Chang, 1990). Antagonist effects of 3,4diaminopyridine on mouse hemidiaphragm muscle, partially paralyzed by the action of nPoly3-APS, further suggest postsynaptic effects. In the range of the concentrations studied, nPoly3-APS also showed a concentration-dependent effect on muscle excitability and excitationcontraction coupling processes, as determined by the observed small blockade of contractile responses by the compound (18.1-54.4 $\mu \mathrm{M})$ upon single and tetanic direct muscle stimulation. nPoly-3-APS has been previously reported to be hemolytic and cytotoxic against different mammalian cell lines (Malovrh et al., 1999) and to produce concentration-dependent reversible/irreversible plasma membrane depolarization in neuronal cells (McClelland et al., 2003). Persistent depolarization may affect muscle excitability and directly-elicited muscle contractility (for review see Ulbricht, 2005). However, our results clearly show that the compound-induced small decrease of directly muscle-elicited twitch and tetanic contraction cannot be explained by a reduction of $\mathrm{rV}_{\mathrm{m}}$.

In conclusion, our results reveal, for the first time, that micromolar concentrations of nPoly-3- 
APS completely inhibit nerve-evoked twitch and tetanic contraction as well as, although partly, directly muscle-elicited twitch and tetanic contraction. No evidence was obtained in the present study for an eventual anticholinesterase effect of nPoly-3-APS. The in vivo toxicity of the natural compound is thus likely to occur through a preferential inhibition of neuromuscular transmission. The most likely mechanistic hypothesis to explain such an inhibition is a slowly reversible antagonist effect on muscle-type nAChRs since nPoly-3-APS reduced the number of open nAChRs by altering neither muscle fiber passive membrane properties nor neurotransmitter ACh release. This blocking $\mathrm{nAChR}$ activity remains however to be confirmed by studying the natural compound effects on muscle-type nAChRs expressed or incorporated in Xenopus oocytes.

\section{Funding information}

This work was supported by Slovenian research grant [P4-0053] and French/Slovenian Proteus grant [BI-FR-PROTEUS/17-18-001, 37446QC].

\section{Compliance with Ethical Standards}

All experiments on isolated tissue followed ethical standards and were approved by The Administration of the Republic of Slovenia for food safety, veterinary and plant protection (3440120/2009/30). The manuscript does not contain clinical studies or patient data.

\section{Conflicts of interest}

The authors declare that there are no conflicts of interest.

\section{Acknowledgments}

The authors gratefully acknowledge American Journal Experts for the English proofreading of the manuscript, and thank Boštjan Drolc and Katarina Babnik for excellent technical support.

\section{References}

Alderdice, M.T., 1982. Further comparison of the effects of physostigmine and neostigmine on frog neuromuscular transmission. Clin. Exp. Pharmacol. Physiol. 9, 35-43. doi: 10.1111/j.1440-1681.1982.tb00776.x

Bunc, M., Sepcic, K., Turk, T., and Suput, D., 2000. In vivo effects of head-to-tail 3alkylpiridinium polymers isolated from the marine sponge Raniera sarai. Pflugers Arch. 440(Suppl 1), R173-R174. doi:10.1007/s004240000052

Catassi, A., Servent, D., Paleari, L., Cesario, A., and Russo, P., 2008. Multiple roles of nicotine on cell proliferation and inhibition of apoptosis: Implications on lung carcinogenesis. Mutat. Res. 659, 221-231. doi: 10.1016/j.mrrev.2008.04.002 
Chang, C.C., Hong, S.J., and Ko, J.L., 1986. Mechanisms of the inhibition by neostigmine of tetanic contraction in the mouse diaphragm. Br. J. Pharmacol. 87, 757-762. doi: 10.1111/j.1476-5381.1986.tb14594.x

Chelossi, E., Mancini, I., Sepcic, K., Turk, T., and Faimali, M., 2006. Comparative antibacterial activity of polymeric 3-alkylpyridinium salts isolated from the Mediterranean sponge Reniera sarai and their synthetic analogues. Biomol. Eng. 23, 317-323. doi: 10.1016/j.bioeng.2006.09.005

Cruz, L.J., Gray, W.R., Olivera, B.M., Zeikus, R.D., Kerr, L., Yoshikami, D., and Moczydlowski, E., 1985. Conus geographus toxins that discriminate between neuronal and muscle sodium channels. J. Biol. Chem. 260, 9280-9288.

Elersek, T., Kosi, G., Turk, T., Pohleven, F., and Sepcic, K., 2008. Influence of polymeric 3alkylpyridinium salts from the marine sponge Reniera sarai on the growth of algae and wood decay fungi. Biofouling 24, 137-143. doi: 10.1080/08927010801935745

Faimali, M., Sepcic, K., Turk, T., and Geraci, S., 2003. Non-toxic antifouling activity of polymeric 3-alkylpyridinium salts from the Mediterranean sponge Reniera sarai (PulitzerFinali). Biofouling 19, 47-56. doi: 10.1080/0892701021000036966

Grandic, M., and Frangez, R., 2014. Pathophysiological effects of synthetic derivatives of polymeric alkylpyridinium salts from the marine sponge, Reniera sarai. Mar. Drugs 12, 2408-2421. doi: 10.3390/md12052408

Grandic, M., Araoz, R., Molgỏ, J., Turk, T., Sepcic, K., Benoit, E., and Frangez, R., 2013. Toxicity of the synthetic polymeric 3-alkylpyridinium salt (APS3) is due to specific block of nicotinic acetylcholine receptors. Toxicology 303, 25-33. doi: 10.1016/j.tox.2012.10.013

Grandic, M., Araoz, R., Molgỏ, J., Turk, T., Sepcic, K., Benoit, E., and Frangez, R., 2012. The non-competitive acetylcholinesterase inhibitor APS12-2 is a potent antagonist of skeletal muscle nicotinic acetylcholine receptors. Toxicol. Appl. Pharmacol. 265, 221-228. doi: 10.1016/j.taap.2012.09.024

Grandic, M., Sepcic, K., Turk, T., Juntes, P., and Frangez, R., 2011. In vivo toxic and lethal cardiovascular effects of a synthetic polymeric 1,3-dodecylpyridinium salt in rodents. Toxicol. Appl. Pharmacol. 255, 86-93. doi: 10.1016/j.taap.2011.06.003

Hong, S.J., and Chang, C.C., 1989. Use of geographutoxin II (mu-conotoxin) for the study of neuromuscular transmission in mouse. Br. J. Pharmacol. 97, 934-940. doi: 10.1111/j.14765381.1989.tb12034.x

Hong, S.J., and Chang, C.C., 1990. Facilitation by 3,4-diaminopyridine of regenerative acetylcholine release from mouse motor nerve. Br. J. Pharmacol. 101, 793-798. doi: $10.1111 /$ j. $1476-5381$

Houssen, W.E., Lu, Z., Edrada-Ebel, R., Chatzi, C., Tucker, S.J., Sepcic, K., Turk, T., Zovko, A., Shen, S., Mancini, I., Scott, R.H., and Jaspars, M., 2010. Chemical synthesis and biological activities of 3-alkyl pyridinium polymeric analogues of marine toxins. J. Chem. Biol. 3, 113-125. doi: 10.1007/s12154-010-0036-4

Katz, B., and Miledi, R., 1973. The binding of acetylcholine to receptors and its removal from 
the synaptic cleft. .J Physiol. 231, 549-574.

Malovrh, P., Sepcic, K., Turk, T., and Macek, P., 1999. Characterization of hemolytic activity of 3-alkylpyridinium polymers from the marine sponge Reniera sarai. Comp. Biochem. Physiol. C Pharmacol. Toxicol. Endocrinol. 124, 221-226. doi: 10.1016/S07428413(99)00067-5

McClelland, D., Evans, R.M., Abidin, I., Sharma, S., Choudhry, F.Z., Jaspars, M., Sepcic, K., and Scott, R.H., 2003. Irreversible and reversible pore formation by polymeric alkylpyridinium salts (poly-APS) from the sponge Reniera sarai. Br. J. Pharmacol. 139, 1399-1408. doi: 10.1038/sj.bjp.0705374

Minic, J., Chatonnet, A., Krejci, E., and Molgó, J., 2003. Butyrylcholinesterase and acetylcholinesterase activity and quantal transmitter release at normal and acetylcholinesterase knockout mouse neuromuscular junctions. Br. J. Pharmacol. 138, 177187. doi: 10.1038/sj.bjp.0705010

Molgỏ, J., Lundh, H., and Thesleff, S., 1980. Potency of 3,4-diaminopyridine and 4aminopyridine on mammalian neuromuscular transmission and the effect of $\mathrm{pH}$ changes. Eur. J. Pharmacol. 61, 25-34. doi: 10.1016/0014-2999(80)90378-7

Paleari, L., Trombino, S., Falugi, C., Gallus, L., Carlone, S., Angelini, C., Sepcic, K., Turk, T., Faimali, M., Noonan, D.M., and Albini, A., 2006. Marine sponge-derived polymeric alkylpyridinium salts as a novel tumor chemotherapeutic targeting the cholinergic system in lung tumors. Int. J. Oncol. 29, 1381-1388. doi: 10.3892/ijo.29.6.1381

Pardo, N.E., Hajela, R.K., and Atchison, W.D., 2006. Acetylcholine release at neuromuscular junctions of adult tottering mice is controlled by $\mathrm{N}$-(cav2.2) and R-type (cav2.3) but not Ltype (cav1.2) Ca2+ channels. J. Pharmacol. Exp. Ther. 319, 1009-1020. doi: 10.1124/jpet.106.108670

Sepcic, K., Guella, G., Mancini, I., Pietra, F., Serra, M.D., Menestrina, G., Tubbs, K., Macek, P., and Turk, T., 1997. Characterization of anticholinesterase-active 3-alkylpyridinium polymers from the marine sponge Reniera sarai in aqueous solutions. J. Nat. Prod. 60, 991996. doi: 10.1021/np970292q

Sepcic, K., Marcel, V., Klaebe, A., Turk, T., Suput, D., and Fournier, D., 1998. Inhibition of acetylcholinesterase by an alkylpyridinium polymer from the marine sponge, Reniera sarai. Biochim. Biophys. Acta. 1387, 217-225. doi: 10.1016/S0167-4838(98)00125-3

Tucker, S.J., McClelland, D., Jaspars, M., Sepcic, K., MacEwan, D.J., and Scott, R.H., 2003. The influence of alkyl pyridinium sponge toxins on membrane properties, cytotoxicity, transfection and protein expression in mammalian cells. Biochim. Biophys. Acta 1614, 171181. doi: 10.1016/S0005-2736(03)00175-5

Turk, T., Frangez, R., and Sepcic, K., 2007. Mechanisms of toxicity of 3-alkylpyridinium polymers from marine sponge Reniera sarai. Mar. Drugs 5, 157-167. doi: 10.3390/MD504157

Ulbricht, W., 2005. Sodium channel inactivation: Molecular determinants and modulation. Physiol. Rev. 85, 1271-1301. doi: 10.1152/physrev.00024.2004 
409 Van der Kloot, W., Balezina, O.P., Molgó, J., and Naves, L.A., 1994. The timing of channel 410 opening during miniature endplate currents at the frog and mouse neuromuscular junctions: 411 effects of fasciculin-2, other anti-cholinesterases and vesamicol. Pflugers Arch. 428, 114-126. 412 doi: 10.1007/BF00374848

413 Wiesner J., Kriz Z., Kuca K., Jun D., Koca J., 2007. Acetylcholinesterases-the structural 414 similarities and differences. J Enzyme Inhib Med Chem. 22, 417-424. doi: $415 \quad 10.1080 / 14756360701421294$

416 Zovko, A., Viktorsson, K., Lewensohn, R., Kolosa, K., Filipic, M., Xing, H., Kem, W.R., 417 Paleari, L., and Turk, T., 2013. APS8, a polymeric alkylpyridinium salt blocks alpha7 nAChR 418 and induces apoptosis in non-small cell lung carcinoma. Mar. Drugs. 11, 2574-2594. doi: $419 \quad 10.3390 / \mathrm{md} 11072574$ 
421 Table 1 Investigated parameters of mouse neuromuscular system in function of protocol stimulation, 422 present results obtained from nPoly-3-APS effects on these parameters, and expected results assuming 423 that the natural compound either inhibits AChE activity or blocks nAChR functioning.

\begin{tabular}{|c|c|c|c|c|}
\hline $\begin{array}{l}\text { Protocol } \\
\text { stimulation }\end{array}$ & Investigated parameters ${ }^{1}$ & Present results ${ }^{1}$ & $\begin{array}{l}\text { Expected results } \\
\text { with nAChR blockage }{ }^{1}\end{array}$ & $\begin{array}{l}\text { Expected results } \\
\text { with } A C h E \text { inhibition }\end{array}$ \\
\hline \multirow{6}{*}{ Nerve } & Twitch amplitude & $\mathbf{Y}$ (important) & $y$ & $\pi$ \\
\hline & Twitch decay phase & $\rightarrow$ & $\rightarrow$ & $\pi$ \\
\hline & Tetanic contraction amplitude & $\mathbf{Y}$ (important) & $y$ & $y$ \\
\hline & Tetanic contraction decay phase & $\rightarrow$ & $\rightarrow$ & $\pi$ \\
\hline & EPP amplitude & $\boldsymbol{\searrow}$ (important) & $y$ & $\pi$ \\
\hline & EPP decay phase & $\rightarrow$ & $\rightarrow$ & $\pi$ \\
\hline \multirow{4}{*}{ Muscle } & Twitch amplitude & $\mathbf{y}$ (small) & $\rightarrow$ & $\rightarrow$ \\
\hline & Twitch decay phase & $\rightarrow$ & $\rightarrow$ & $\rightarrow$ \\
\hline & Tetanic contraction amplitude & $\searrow$ (small) & $\rightarrow$ & $\rightarrow$ \\
\hline & Tetanic contraction decay phase & $\rightarrow$ & $\rightarrow$ & $\rightarrow$ \\
\hline \multirow{4}{*}{ None } & MEPP amplitude & $\mathbf{Y}$ (important) & $y$ & $\pi$ \\
\hline & MEPP frequency & $\searrow$ (high concentrations) & $y$ & $\pi$ \\
\hline & MEPP decay phase & $\rightarrow$ & $\rightarrow$ & $\pi$ \\
\hline & Resting membrane potential & $\rightarrow$ & $\rightarrow$ & $y$ \\
\hline
\end{tabular}

$424{ }^{1}$ Specific effects due to nAChR blockage are underline in grey.

425 

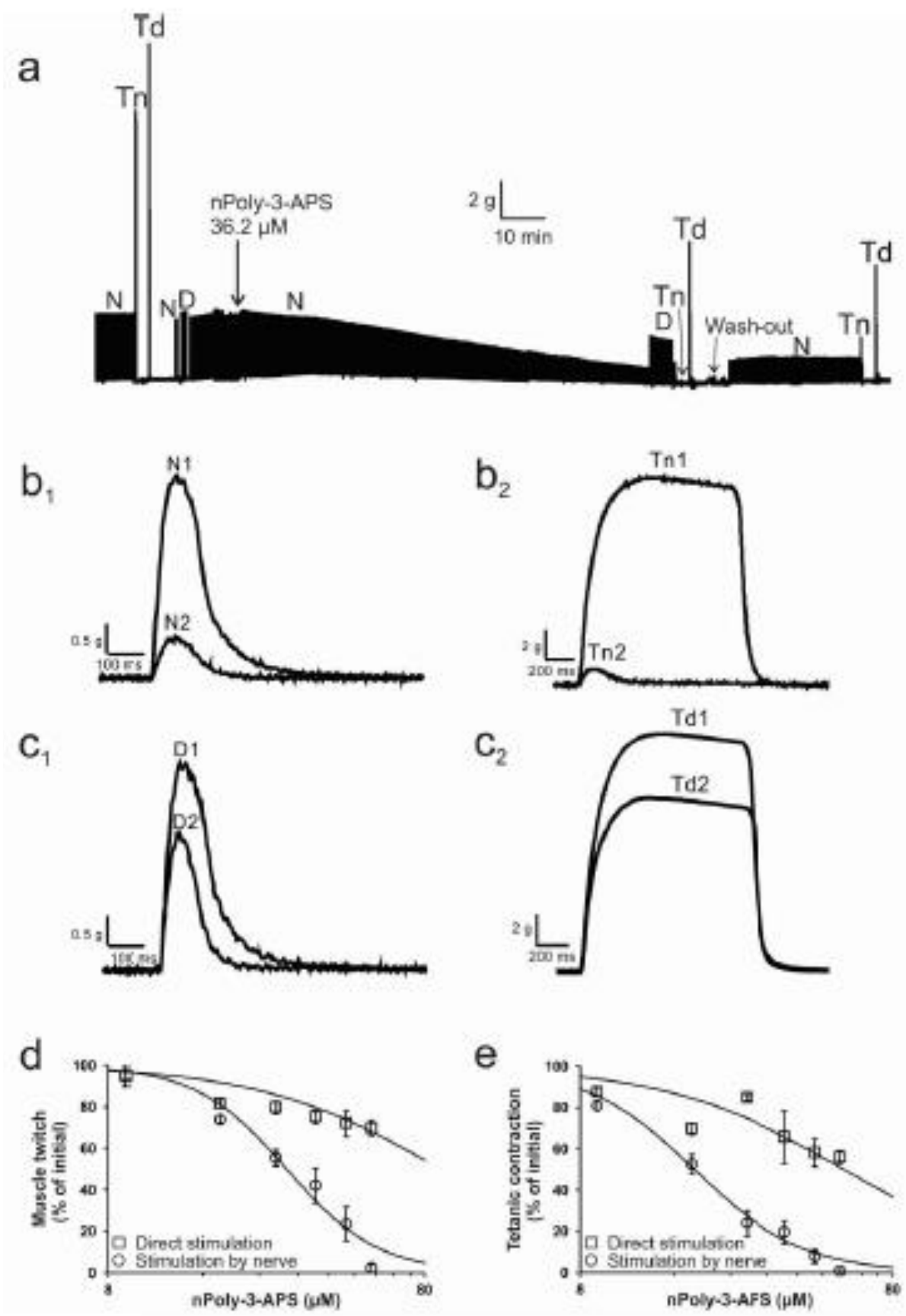

Fig 1 Effects of nPoly-3-APS on nerve-evoked and directly muscle-elicited twitch and tetanic contraction in isolated mouse hemidiaphragm neuromuscular preparations. (a) Representative time-course of nPoly-3-APS $(36.2 \mu \mathrm{M})$ effects on muscle contraction showing the partial and reversible block of nerve-evoked and directly muscle-elicited twitch and tetanic contraction induced by the compound. $\mathrm{N}$ denotes nerve-evoked muscle twitch; $\mathrm{D}$ denotes directly muscleelicited twitch; Tn denotes nerve-evoked tetanic contraction; Td denotes directly muscleelicited tetanic contraction. (b1) Traces of nerve-evoked twitch recorded before (N1) and 90 min after (N2) the application of $36.2 \mu \mathrm{M}$ nPoly-3-APS. (b2) Traces of tetanic contraction recorded before (Tn1) and $90 \mathrm{~min}$ after (Tn2) the application of $36.2 \mu \mathrm{M}$ nPoly-3-APS. (c1) Representative recordings of twitch elicited by direct muscle stimulation, before (D1) and 90 min after (D2) the application of $36.2 \mu \mathrm{M}$ nPoly-3-APS. (c2) Representative recordings of 
439 tetanic contraction elicited by direct muscle stimulation, before (Td1) and 90 min after (Td2)

440 the application of $36.2 \mu \mathrm{M}$ nPoly-3-APS. (d) Concentration-response curves for the inhibition

441 of directly muscle-elicited and nerve-evoked twitch by nPoly-3-APS. Muscle twitch was

442 expressed as percentage of its maximal initial value. Each value represents the mean \pm S.E.

443 obtained from five different muscles. $\mathrm{IC}_{50}$ values, determined by fitting the relationships with

444 the four-parameter nonlinear regression model, were 29.4 and $90 \mu \mathrm{M}$ for nerve-evoked and

445 directly muscle-elicited twitches, respectively $\left(r^{2} \geq 0.993\right)$. (e) Concentration-response curves

446 for the inhibition of directly muscle-elicited and nerve-evoked tetanic contraction by nPoly-3-

447 APS. Tetanic contraction was expressed as percentage of its maximal initial value. Each value

448 represents the mean \pm S.E. obtained from five different muscles. $\mathrm{IC}_{50}$ values, determined by

449 fitting the relationships with the four-parameter nonlinear regression model, were 18.5 and 56

$450 \mu \mathrm{M}$ for nerve-evoked and directly muscle-elicited tetanic contractions, respectively $\left(\mathrm{r}^{2} \geq\right.$ $4510.978)$

452 


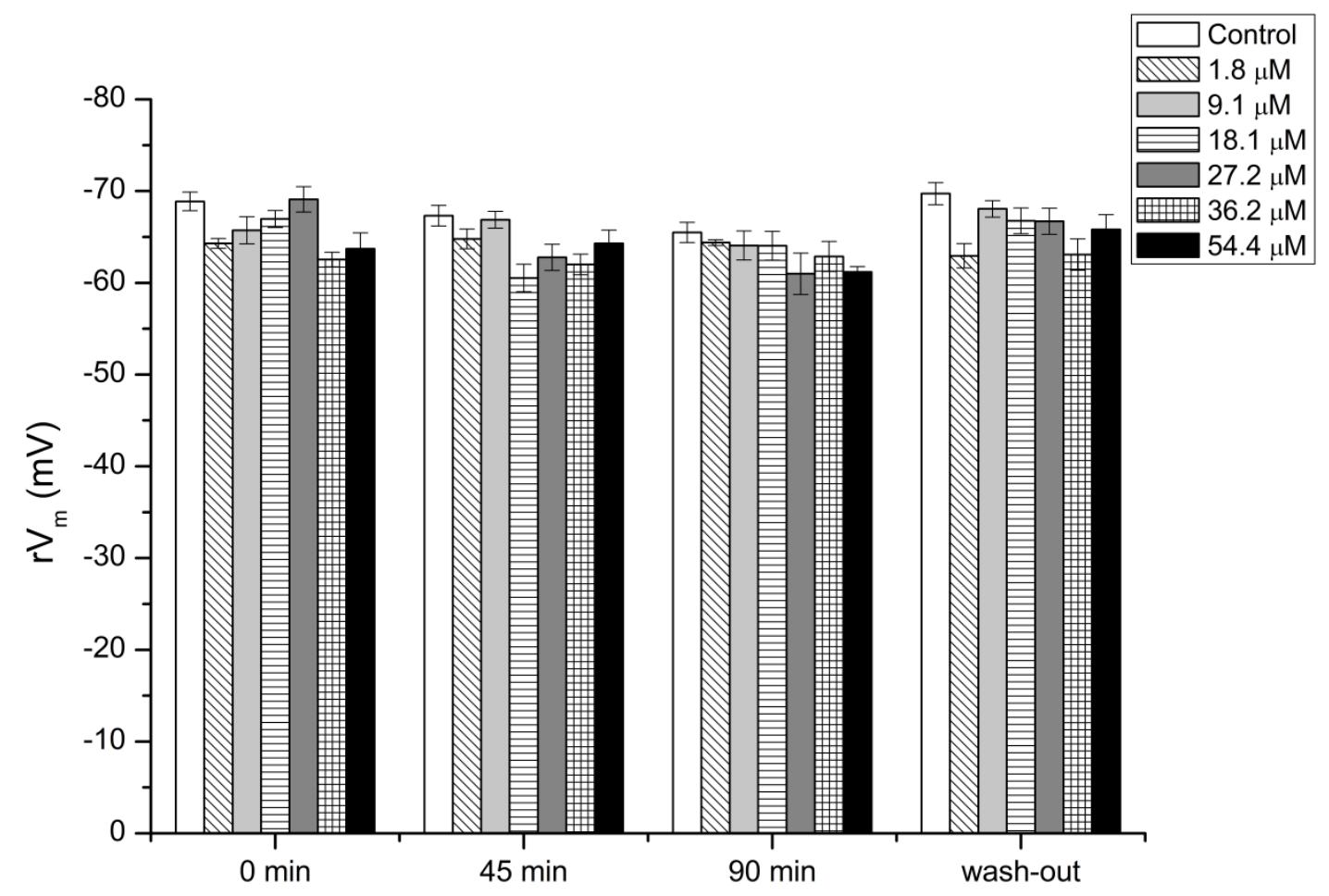

454

455 Fig 2 Concentration- and time-dependent effect of nPoly-3-APS on the muscle fiber resting 456 membrane potential $\left(\mathrm{rV}_{\mathrm{m}}\right)$ of the mouse hemidiaphragm. Neuromuscular preparations were 457 exposed to 1.8-54.4 $\mu \mathrm{M}$ nPoly-3-APS. Measurements were performed before (time 0 ), 45 and $45890 \mathrm{~min}$ after nPoly-3-APS application, and after about $30 \mathrm{~min}$ of thorough washing of the 459 preparation with standard physiological solution. Each value represents the mean \pm S.E. of $460 \quad \mathrm{rV}_{\mathrm{m}}$ recorded from 3 different hemidiaphragms $(\mathrm{n}=7-10$ fibers from each) 
a

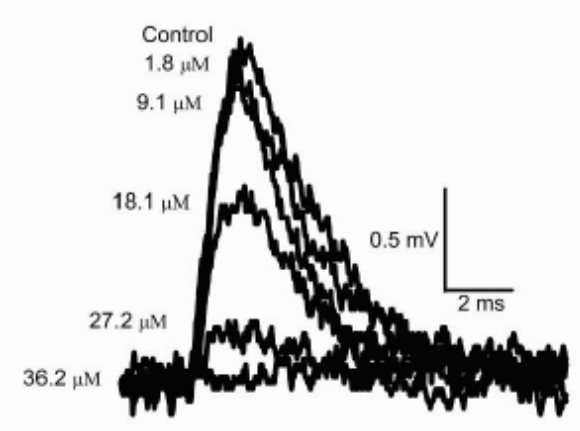

C

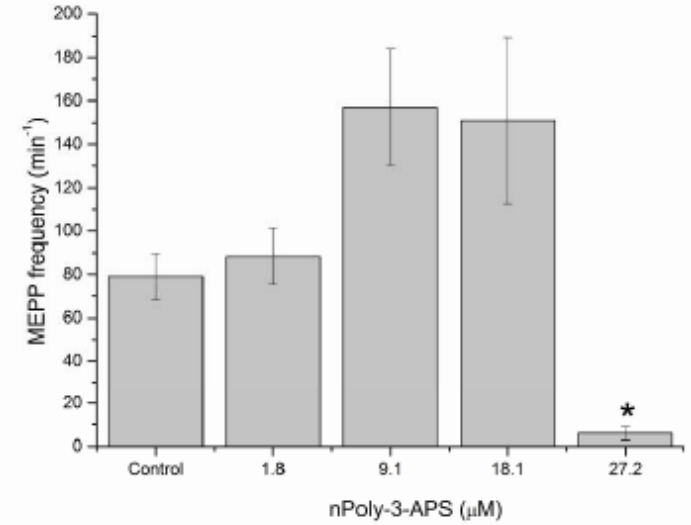

b

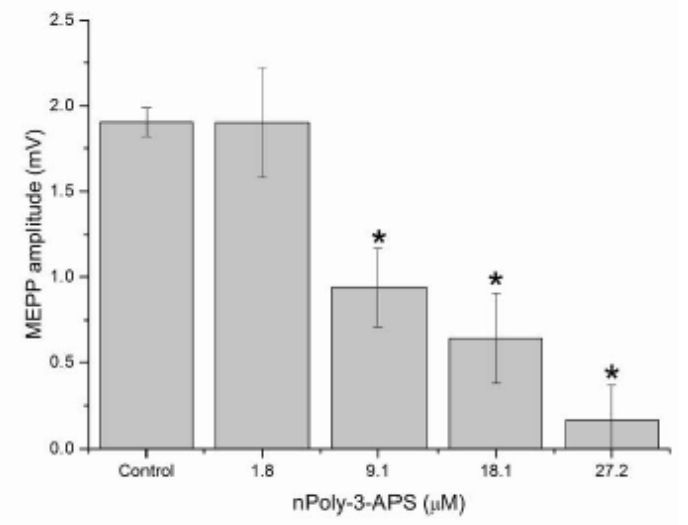

462

463

464

465

466

467

468

469

470

471

Fig 3 Effects of nPoly-3-APS on the MEPP amplitude and frequency in the muscle fibers of isolated mouse hemidiaphragm. (a) MEPP traces recorded under control conditions and after the indicated concentrations of nPoly-3-APS applied for $90 \mathrm{~min}$. At $36.2 \mu \mathrm{M}$, the compound completely blocked MEPPs. (b) MEPP amplitude and (c) MEPP frequency in muscle fibers exposed to different nPoly-3-APS concentrations. Each bar represents the mean value \pm S.E. from 3 different hemidiaphragms $(n=7-10$ fibers from each). $*$ indicates statistically significant difference $(P \leq 0.05)$ 
a

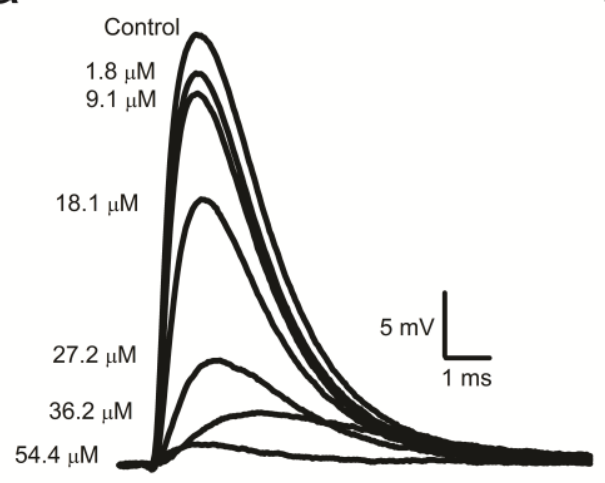

b

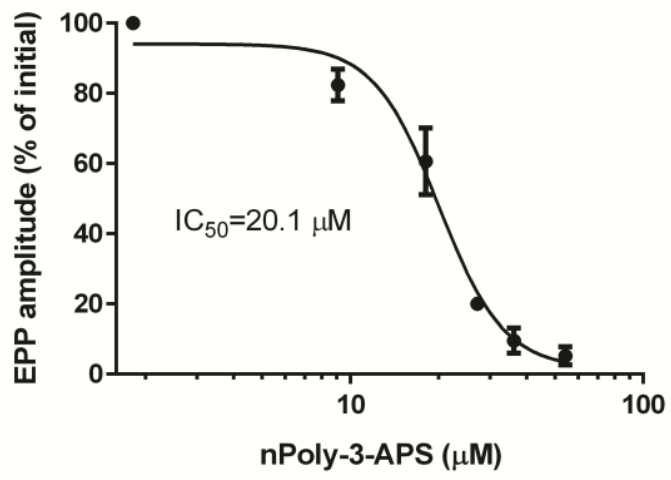

473

474 Fig 4 Effects of nPoly-3-APS on EPP amplitude in mouse hemidiaphragm skeletal muscle

475 fibers. The compound blocked EPPs in a concentration-dependent manner. (a) EPP traces

476 recorded under control conditions and $90 \mathrm{~min}$ after exposure of muscle fibers to various

477 nPoly-3-APS concentrations. Note that the decrease of EPP amplitudes occurred without 478 significant change in $\mathrm{rV}_{\mathrm{m}}$. (b) Concentration-response curve for the inhibition of EPP 479 amplitude, expressed as a percentage of its maximal initial value, by nPoly-3-APS3. Each 480 value represents the mean \pm S.E. of EPPs recorded from at least 3 different hemidiaphragms 481 ( $\mathrm{n}=7-10$ muscle fibers from each). The $\mathrm{IC}_{50}$ value, determined by fitting the relationship with 482 the four-parameter nonlinear regression model, was $20.1 \mu \mathrm{M}\left(\mathrm{r}^{2}=0.985\right)$ 